\title{
Differentiable Functions into Real Normed Spaces
}

\author{
Hiroyuki Okazaki \\ Shinshu University \\ Nagano, Japan
}

Keiko Narita

Hirosaki-city

Aomori, Japan

\author{
Noboru Endou \\ Nagano National College of Technology \\ Nagano, Japan \\ Yasunari Shidama \\ Shinshu University \\ Nagano, Japan
}

Summary. In this article, we formalize the differentiability of functions from the set of real numbers into a normed vector space [14].

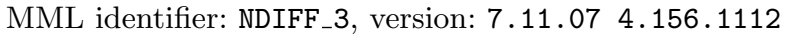

The notation and terminology used here have been introduced in the following papers: [12], [2], [3], [7], [9], [11], [1], [4], [10], [13], [6], [17], [18], [15], [8], [16], [19], and [5].

For simplicity, we adopt the following rules: $F$ denotes a non trivial real normed space, $G$ denotes a real normed space, $X$ denotes a set, $x, x_{0}, r, p$ denote real numbers, $n, k$ denote elements of $\mathbb{N}, Y$ denotes a subset of $\mathbb{R}, Z$ denotes an open subset of $\mathbb{R}, s_{1}$ denotes a sequence of real numbers, $s_{2}$ denotes a sequence of $G, f, f_{1}, f_{2}$ denote partial functions from $\mathbb{R}$ to the carrier of $F$, $h$ denotes a convergent to 0 sequence of real numbers, and $c$ denotes a constant sequence of real numbers.

We now state two propositions:

(1) If for every $n$ holds $\left\|s_{2}(n)\right\| \leq s_{1}(n)$ and $s_{1}$ is convergent and $\lim s_{1}=0$, then $s_{2}$ is convergent and $\lim s_{2}=0_{G}$.

(2) $\quad\left(s_{1} \uparrow k\right)\left(s_{2} \uparrow k\right)=\left(s_{1} s_{2}\right) \uparrow k$.

Let us consider $F$ and let $I_{1}$ be a partial function from $\mathbb{R}$ to the carrier of $F$. We say that $I_{1}$ is rest-like if and only if: 
(Def. 1) $I_{1}$ is total and for every $h$ holds $h^{-1}\left(I_{1 *} h\right)$ is convergent and $\lim \left(h^{-1}\left(I_{1 *} h\right)\right)=0_{F}$.

Let us consider $F$. One can check that there exists a partial function from $\mathbb{R}$ to the carrier of $F$ which is rest-like. Let us consider $F$. A rest of $F$ is a rest-like partial function from $\mathbb{R}$ to the carrier of $F$. Let us consider $F$ and let $I_{1}$ be a function from $\mathbb{R}$ into the carrier of $F$. We say that $I_{1}$ is linear if and only if:

(Def. 2) There exists a point $r$ of $F$ such that for every real number $p$ holds $I_{1}(p)=p \cdot r$.

Let us consider $F$. Note that there exists a function from $\mathbb{R}$ into the carrier of $F$ which is linear. Let us consider $F$. A linear of $F$ is a linear function from $\mathbb{R}$ into the carrier of $F$.

We use the following convention: $R, R_{1}, R_{2}$ denote rests of $F$ and $L, L_{1}, L_{2}$ denote linears of $F$.

The following propositions are true:

(3) $L_{1}+L_{2}$ is a linear of $F$ and $L_{1}-L_{2}$ is a linear of $F$.

(4) $r L$ is a linear of $F$.

(5) Let $h_{1}, h_{2}$ be partial functions from $\mathbb{R}$ to the carrier of $F$ and $s_{2}$ be a sequence of real numbers. If rng $s_{2} \subseteq \operatorname{dom} h_{1} \cap \operatorname{dom} h_{2}$, then $\left(h_{1}+h_{2}\right)_{*} s_{2}=$ $\left(h_{1 *} s_{2}\right)+\left(h_{2 *} s_{2}\right)$ and $\left(h_{1}-h_{2}\right)_{*} s_{2}=\left(h_{1 *} s_{2}\right)-\left(h_{2 *} s_{2}\right)$.

(6) Let $h_{1}, h_{2}$ be partial functions from $\mathbb{R}$ to the carrier of $F$ and $s_{2}$ be a sequence of real numbers. If $h_{1}$ is total and $h_{2}$ is total, then $\left(h_{1}+h_{2}\right)_{*} s_{2}=$ $\left(h_{1 *} s_{2}\right)+\left(h_{2 *} s_{2}\right)$ and $\left(h_{1}-h_{2}\right)_{*} s_{2}=\left(h_{1 *} s_{2}\right)-\left(h_{2 *} s_{2}\right)$.

(7) $R_{1}+R_{2}$ is a rest of $F$ and $R_{1}-R_{2}$ is a rest of $F$.

(8) $r R$ is a rest of $F$.

Let us consider $F, f$ and let $x_{0}$ be a real number. We say that $f$ is differentiable in $x_{0}$ if and only if:

(Def. 3) There exists a neighbourhood $N$ of $x_{0}$ such that $N \subseteq \operatorname{dom} f$ and there exist $L, R$ such that for every $x$ such that $x \in N$ holds $f_{x}-f_{x_{0}}=L(x-$ $\left.x_{0}\right)+R_{x-x_{0}}$.

Let us consider $F, f$ and let $x_{0}$ be a real number. Let us assume that $f$ is differentiable in $x_{0}$. The functor $f^{\prime}\left(x_{0}\right)$ yielding a point of $F$ is defined by the condition (Def. 4).

(Def. 4) There exists a neighbourhood $N$ of $x_{0}$ such that $N \subseteq \operatorname{dom} f$ and there exist $L, R$ such that $f^{\prime}\left(x_{0}\right)=L(1)$ and for every $x$ such that $x \in N$ holds $f_{x}-f_{x_{0}}=L\left(x-x_{0}\right)+R_{x-x_{0}}$.

Let us consider $F, f, X$. We say that $f$ is differentiable on $X$ if and only if:

(Def. 5) $X \subseteq \operatorname{dom} f$ and for every $x$ such that $x \in X$ holds $f \uparrow X$ is differentiable in $x$.

The following propositions are true: 
(9) If $f$ is differentiable on $X$, then $X$ is a subset of $\mathbb{R}$.

(10) $f$ is differentiable on $Z$ iff $Z \subseteq \operatorname{dom} f$ and for every $x$ such that $x \in Z$ holds $f$ is differentiable in $x$.

(11) If $f$ is differentiable on $Y$, then $Y$ is open.

Let us consider $F, f, X$. Let us assume that $f$ is differentiable on $X$. The functor $f_{\lceil X}^{\prime}$ yields a partial function from $\mathbb{R}$ to the carrier of $F$ and is defined by:

(Def. 6) $\operatorname{dom}\left(f_{\lceil X}^{\prime}\right)=X$ and for every $x$ such that $x \in X$ holds $f_{\lceil X}^{\prime}(x)=f^{\prime}(x)$.

Next we state a number of propositions:

(12) Suppose $Z \subseteq \operatorname{dom} f$ and there exists a point $r$ of $F$ such that $\operatorname{rng} f=\{r\}$. Then $f$ is differentiable on $Z$ and for every $x$ such that $x \in Z$ holds $\left(f_{\uparrow Z}^{\prime}\right)_{x}=0_{F}$.

(13) Let $x_{0}$ be a real number and $N$ be a neighbourhood of $x_{0}$. Suppose $f$ is differentiable in $x_{0}$ and $N \subseteq \operatorname{dom} f$. Let given $h, c$. Suppose $\operatorname{rng} c=\left\{x_{0}\right\}$ and $\operatorname{rng}(h+c) \subseteq N$. Then $h^{-1}\left(\left(f_{*}(h+c)\right)-\left(f_{*} c\right)\right)$ is convergent and $f^{\prime}\left(x_{0}\right)=\lim \left(h^{-1}\left(\left(f_{*}(h+c)\right)-\left(f_{*} c\right)\right)\right)$.

(14) If $f_{1}$ is differentiable in $x_{0}$ and $f_{2}$ is differentiable in $x_{0}$, then $f_{1}+f_{2}$ is differentiable in $x_{0}$ and $\left(f_{1}+f_{2}\right)^{\prime}\left(x_{0}\right)=f_{1}{ }^{\prime}\left(x_{0}\right)+f_{2}{ }^{\prime}\left(x_{0}\right)$.

(15) If $f_{1}$ is differentiable in $x_{0}$ and $f_{2}$ is differentiable in $x_{0}$, then $f_{1}-f_{2}$ is differentiable in $x_{0}$ and $\left(f_{1}-f_{2}\right)^{\prime}\left(x_{0}\right)=f_{1}{ }^{\prime}\left(x_{0}\right)-f_{2}{ }^{\prime}\left(x_{0}\right)$.

(16) For every real number $r$ such that $f$ is differentiable in $x_{0}$ holds $r f$ is differentiable in $x_{0}$ and $(r f)^{\prime}\left(x_{0}\right)=r \cdot f^{\prime}\left(x_{0}\right)$.

(17) Suppose $Z \subseteq \operatorname{dom}\left(f_{1}+f_{2}\right)$ and $f_{1}$ is differentiable on $Z$ and $f_{2}$ is differentiable on $Z$. Then $f_{1}+f_{2}$ is differentiable on $Z$ and for every $x$ such that $x \in Z$ holds $\left(f_{1}+f_{2}\right)^{\prime}{ }_{\uparrow Z}(x)=f_{1}{ }^{\prime}(x)+{f_{2}}^{\prime}(x)$.

(18) Suppose $Z \subseteq \operatorname{dom}\left(f_{1}-f_{2}\right)$ and $f_{1}$ is differentiable on $Z$ and $f_{2}$ is differentiable on $Z$. Then $f_{1}-f_{2}$ is differentiable on $Z$ and for every $x$ such that $x \in Z$ holds $\left(f_{1}-f_{2}\right)^{\prime}{ }_{\mid Z}(x)=f_{1}{ }^{\prime}(x)-f_{2}{ }^{\prime}(x)$.

(19) Suppose $Z \subseteq \operatorname{dom}(r f)$ and $f$ is differentiable on $Z$. Then $r f$ is differentiable on $Z$ and for every $x$ such that $x \in Z$ holds $(r f)^{\prime}{ }_{\mid Z}(x)=r \cdot f^{\prime}(x)$.

(20) If $Z \subseteq \operatorname{dom} f$ and $f \nmid Z$ is constant, then $f$ is differentiable on $Z$ and for every $x$ such that $x \in Z$ holds $f_{\uparrow Z}^{\prime}(x)=0_{F}$.

(21) Let $r, p$ be points of $F$ and given $Z, f$. Suppose $Z \subseteq \operatorname{dom} f$ and for every $x$ such that $x \in Z$ holds $f_{x}=x \cdot r+p$. Then $f$ is differentiable on $Z$ and for every $x$ such that $x \in Z$ holds $f_{\uparrow Z}^{\prime}(x)=r$.

(22) For every real number $x_{0}$ such that $f$ is differentiable in $x_{0}$ holds $f$ is continuous in $x_{0}$.

(23) If $f$ is differentiable on $X$, then $f\lceil X$ is continuous.

(24) If $f$ is differentiable on $X$ and $Z \subseteq X$, then $f$ is differentiable on $Z$. 
(25) There exists a rest $R$ of $F$ such that $R_{0}=0_{F}$ and $R$ is continuous in 0 .

Let us consider $F$ and let $f$ be a partial function from $\mathbb{R}$ to the carrier of $F$. We say that $f$ is differentiable if and only if:

(Def. 7) $f$ is differentiable on $\operatorname{dom} f$.

Let us consider $F$. One can check that there exists a function from $\mathbb{R}$ into the carrier of $F$ which is differentiable. We now state the proposition

(26) Let $f$ be a differentiable partial function from $\mathbb{R}$ to the carrier of $F$. If $Z \subseteq \operatorname{dom} f$, then $f$ is differentiable on $Z$.

\section{REFERENCES}

[1] Grzegorz Bancerek. The ordinal numbers. Formalized Mathematics, 1(1):91-96, 1990.

[2] Czesław Byliński. Functions and their basic properties. Formalized Mathematics, 1(1):5565, 1990.

[3] Czesław Byliński. Functions from a set to a set. Formalized Mathematics, 1(1):153-164, 1990.

[4] Czesław Byliński. Partial functions. Formalized Mathematics, 1(2):357-367, 1990.

[5] Czesław Byliński. Some basic properties of sets. Formalized Mathematics, 1(1):47-53, 1990.

[6] Krzysztof Hryniewiecki. Basic properties of real numbers. Formalized Mathematics, 1(1):35-40, 1990.

[7] Hiroshi Imura, Morishige Kimura, and Yasunari Shidama. The differentiable functions on normed linear spaces. Formalized Mathematics, 12(3):321-327, 2004.

[8] Jarosław Kotowicz. Real sequences and basic operations on them. Formalized Mathematics, 1(2):269-272, 1990.

[9] Hiroyuki Okazaki, Noboru Endou, and Yasunari Shidama. More on continuous functions on normed linear spaces. Formalized Mathematics, 19(1):45-49, 2011, doi: 10.2478/v10037-011-0008-3.

[10] Beata Padlewska and Agata Darmochwał. Topological spaces and continuous functions. Formalized Mathematics, 1(1):223-230, 1990.

[11] Jan Popiołek. Real normed space. Formalized Mathematics, 2(1):111-115, 1991.

[12] Konrad Raczkowski and Paweł Sadowski. Real function differentiability. Formalized Mathematics, 1(4):797-801, 1990.

[13] Konrad Raczkowski and Paweł Sadowski. Topological properties of subsets in real numbers. Formalized Mathematics, 1(4):777-780, 1990.

[14] Laurent Schwartz. Cours d'analyse, vol. 1. Hermann Paris, 1967.

[15] Wojciech A. Trybulec. Vectors in real linear space. Formalized Mathematics, 1(2):291-296, 1990.

[16] Zinaida Trybulec. Properties of subsets. Formalized Mathematics, 1(1):67-71, 1990.

[17] Edmund Woronowicz. Relations and their basic properties. Formalized Mathematics, 1(1):73-83, 1990.

[18] Edmund Woronowicz. Relations defined on sets. Formalized Mathematics, 1(1):181-186, 1990.

[19] Hiroshi Yamazaki and Yasunari Shidama. Algebra of vector functions. Formalized Mathematics, 3(2):171-175, 1992. 